\title{
Pathologic findings of Fabry nephropathy: the pivotal role of kidney biopsy
}

\author{
Jung-ho Shin, Su Hyun Kim \\ Division of Nephrology, Department of Internal Medicine, Chung-Ang University Hospital, Seoul, Republic of Korea
}

\section{See Article on Page 611-619}

Fabry disease is a rare lysosomal storage disorder caused by absent or reduced activity of $\alpha$-galactosidase $\mathrm{A}$, and the subsequent systemic accumulation of predominantly globotriaosylceramide (GL3, also called GB3) is caused by mutations in the a-gal A-encoding gene on the $\mathrm{X}$ chromosome (Xq22.1). This deposition within the lysosomes triggers pathogenic pathways in the vascular endothelium and activities of cells of different tissues in vital organs (renal, cardiac, and nervous systems) that lead to cell death and irreversible organ damage.

The severity of clinical expression in hemizygous males correlates with a-gal residual activity, and the classical phenotype develops in individuals with $<1 \%$ of residual enzyme activity. At a young age, classic male patients show severe general symptoms (neuropathic pain, angiokeratoma, hypohidrosis, and corneal opacity). Kidney involvement is a significant feature of Fabry disease, and untreated classical male patients develop end-stage renal disease in the third to fifth decade of life. On the other hand, manifestations of heterozygous females can range from asymptom- atic, mild, or severe due to skewed X inactivation [1].

Fabry disease can be divided into a classic phenotype and a late-onset variant (nonclassical or atypical). This clinical phenotype is usually considered to be defined (at least partially) by the genotype. Classic Fabry disease manifests with typical symptoms of absent or low enzyme activity levels that begin in childhood. Late-onset Fabry disease is characterized by a more variable disease course (adulthood onset) with residual enzyme activity. In this issue of Kidney Research and Clinical Practice, Kim et al. [2] report the clinical and pathologic findings of patients with Fabry disease who underwent kidney biopsy before or after enzyme replacement therapy [ERT]. In particular, the before-treatment group showed pathologic GL3 accumulation in kidney tissues, even in those without microalbuminuria. Apparent nephropathy, including GL3 accumulation, can occur in patients with normal glomerular filtration rate and no or minimal microalbuminuria.

In a recent study in 14 patients with Fabry disease between 4 and 19 years of age with normal glomerular filtration rates, the amount of GL3 accumulation in the podocytes correlated with age [3]. In a study by Tøndel et al. [4], loss of segmental foot processes was observed in patients

Received: October 19, 2021; Revised: October 22, 2021; Accepted: October 22, 2021

Editor: Tae-Hyun Yoo, Yonsei University, Seoul, Republic of Korea

Correspondence: Su Hyun Kim

Division of Nephrology, Department of Internal Medicine, Chung-Ang University Hospital, 102 Heukseok-ro, Dongjak-gu, Seoul 06973, Republic of Korea.E-mail:sh76so@cau.ac.kr

ORCID: https://orcid.org/0000-0003-3382-528X

(a) This is an Open Access article distributed under the terms of the Creative Commons Attribution Non-Commercial and No Derivatives License (http:// creativecommons.org/licenses/by-nc-nd/4.0/) which permits unrestricted non-commercial use, distribution of the material without any modifications, and reproduction in any medium, provided the original works properly cited. 


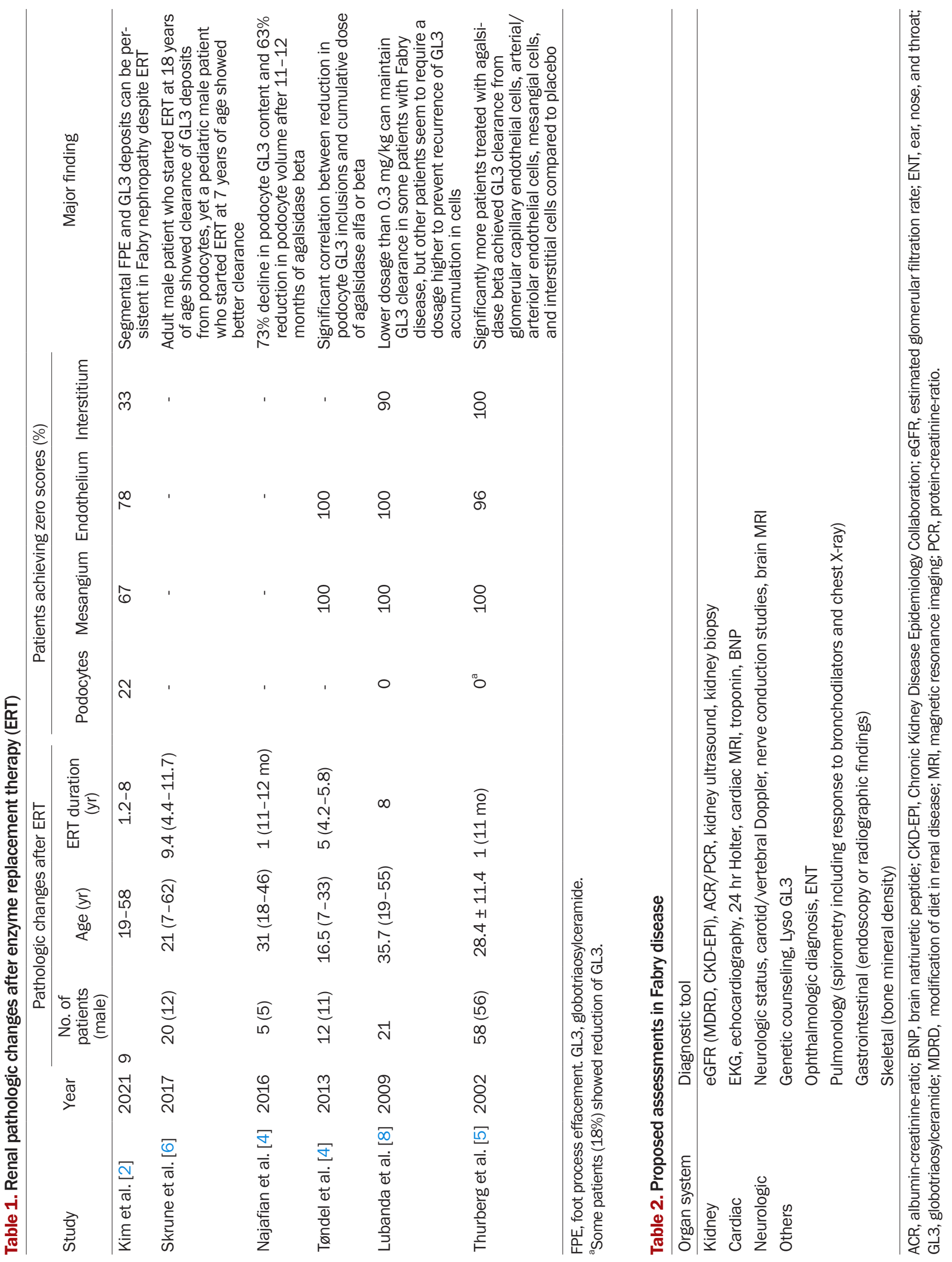


with Fabry disease with albuminuria in the normal range of less than $30 \mathrm{mg} /$ day. Similarly, the present study showed that podocyte foot process effacement (FPE) is one of the earliest signs of renal damage in Fabry disease.

Thurberg et al. [5] reported that patients receiving 11 months of ERT demonstrated a 100\% reduction (complete clearance) in GL3 in peritubular capillary endothelial, mesangial, and interstitial cells. In contrast, only $18 \%$ of patients showed a reduction of GL3 in podocytes that responded after 11 months of ERT. This result might reflect the rate at which these cells turn over. In contrast to endothelial and mesangial cells, podocytes are differentiated terminally and proliferate poorly in response to injury or loss. We summarized several studies of renal pathologic changes after ERT for patients with Fabry disease (Table 1) [2,4-8]. Higher dose and early initiation of ERT might be positively associated with clearance of GL3 deposits from podocytes $[4,6,8]$.

For assessment of disease severity and renal effect of ERT, we recommend histopathologic examination of the glomerular, tubulointerstitial, and vascular compartments. Therefore, kidney biopsy is pivotal. Because it can serve as a standard for treatment, kidney biopsy is a crucial screening test for women who suffer from classical complications. Therefore, kidney biopsy can be performed in a Fabry disease patient group to assess the degree of damage to the underlying tissues before enzymatic treatment and for clinical judgment of Fabry disease with atypical expression. ERT leads to a rapid and marked decrease in mesangial and endothelial cell GL3 inclusions, whereas podocyte inclusions and proteinuria persist despite treatment. Early intervention is crucial because ERT is less effective in more advanced diseases and results in irreversible damage.

Since Fabry disease is a progressive multisystem disease, various organs should be tested upon diagnosis (Table 2) $[9,10]$. A renal examination can be performed by a nephrologist (including estimated glomerular filtration rate and albuminuria), but involvement of other vital organs (especially the cardiac and neurologic) must be determined by an appropriate specialist.

In summary, Kim et al. [2] suggest the pivotal role of kidney biopsy in patients with Fabry disease as a screening tool for kidney damage and as an ERT response evaluation tool. In addition, they demonstrate segmental FPE and GL3 accumulation in renal pathologic findings even in patients with normoalbuminuria. Kidney biopsy is a vital tool in assessing renal involvement and can lead to early initiation of ERT, which can change the course of Fabry disease.

\section{Conflicts of interest}

All authors have no conflicts of interest to declare.

\section{Authors' contributions}

Writing-original draft: All authors

All authors read and approved the final manuscript.

\section{ORCID}

Jung-ho Shin, https://orcid.org/0000-0001-9755-3100

Su Hyun Kim, https://orcid.org/0000-0003-3382-528X

\section{References}

1. Echevarria L, Benistan K, Toussaint A, et al. X-chromosome inactivation in female patients with Fabry disease. Clin Genet 2016;89:44-54.

2. Kim IY, Lee HJ, Cheon CK. Fabry nephropathy before and after enzyme replacement therapy: important role of renal biopsy in patients with Fabry disease. Kidney Res Clin Pract 2021;40:611619.

3. Najafian B, Tøndel C, Svarstad E, Gubler MC, Oliveira JP, Mauer M. Accumulation of globotriaosylceramide in podocytes in Fabry nephropathy is associated with progressive podocyte loss. $J$ Am Soc Nephrol 2020;31:865-875.

4. Tøndel C, Bostad L, Larsen KK, et al. Agalsidase benefits renal histology in young patients with Fabry disease. J Am Soc Nephrol 2013;24:137-148.

5. Thurberg BL, Rennke H, Colvin RB, et al. Globotriaosylceramide accumulation in the Fabry kidney is cleared from multiple cell types after enzyme replacement therapy. Kidney Int 2002;62:1933-1946.

6. Skrunes R, Tøndel C, Leh S, et al. Long-term dose-dependent agalsidase effects on kidney histology in Fabry disease. Clin J Am Soc Nephrol 2017;12:1470-1479.

7. Najafian B, Tøndel C, Svarstad E, Sokolovkiy A, Smith K, Mauer M. One year of enzyme replacement therapy reduces globotriaosylceramide inclusions in podocytes in male adult patients with Fabry disease. PLoS One 2016;11:e0152812. 
8. Lubanda JC, Anijalg E, Bzdúch V, Thurberg BL, Bénichou B, Tylki-Szymanska A. Evaluation of a low dose, after a standard therapeutic dose, of agalsidase beta during enzyme replacement therapy in patients with Fabry disease. Genet Med 2009;11:256264.
9. Cairns T, Müntze J, Gernert J, Spingler L, Nordbeck P, Wanner C. Hot topics in Fabry disease. Postgrad Med J 2018;94:709-713.

10. Silva CA, Moura-Neto JA, Dos Reis MA, Vieira Neto OM, Barreto FC. Renal manifestations of Fabry disease: a narrative review. Can J Kidney Health Dis 2021;8:2054358120985627. 